\title{
An Exploratory Study on the Adaptive Word-of-Mouth Communication in Seeker-initiated Context
}

\author{
Cheng-Hsi Fang \\ Dept. of Marketing and Distribution, Chien-Hsin University of Science and Technology \\ 229, Chien-Hsin Rd., Jung-Li 320, Taiwan \\ Tel: 886-458-1196 ext 7512 E-mail: budafang@gmail.com
}

Received: December 8, 2012 Accepted: January 9, 2013 Published: June 26, 2013

doi:10.5296/bms.v4i1.3913 URL: http://dx.doi.org/10.5296/bms.v4i1.3913

\begin{abstract}
The purpose of this research was to examine the strategies adopted by the word of mouth (WOM) dyad in seeker-initiated context. To examine this process, in-depth interviews were conducted with 14 dyads. The result indicated that the WOM sender has two special motivations, risk aversion and egotism, found only in seeker-initiated WOM context. Since spreading WOM is sometimes a risk taking behavior, the sender uses different strategies in response to the seeker's request. A preliminary model of this adaptive WOM communication was presented. The results can help us clarify the interaction between WOM dyad; moreover, it provides another perspective to look at this complicated and multifaceted behavior.
\end{abstract}

Keywords: Referral, Risk aversion, Regret, Buzz, Social interaction 


\section{Introduction}

Consumer research has traditionally viewed WOM as self-evident, assuming that it involves only messages exchanged among and between individual customers. However, when researchers examined the power of WOM, they actually meant at least two different types of behavior. First, based on the information sharing theory, people may actively share information with their friends to help them make a decision or to signal their wisdom (Hennig-Thurau et al., 2004). This sender-initiated WOM is the key successful factor for many fads or popular goods; for example, the Harry Potter book series (Cohen, 1999) and the Endnote software (Rosen, 2000) in the academic society. One of the major issues in this research stream is to identify the WOM sender, such as opinion leaders (Rogers, 1983) or market mavens (Feick \& Price, 1987).

Second, WOM may be triggered by receiver's request. Especially when individuals experience a high degree of uncertainty about a particular buying situation, they usually ask their friends for product opinions or even delegate the entire purchase decision to their friends (Aggarwal \& Mazumdar, 2008). According to Mangold, Miller, and Brockway's (1999) study, the seeker initiated over 50\% of the WOM incidents. However, it is somewhat surprising that little research examined the effect of WOM in this situation. Besides, to our knowledge, none of the past studies tried to distinguish different effects of the seeker-initiated WOM and the sender-initiated WOM. When people actively acquire WOM references, they are largely influenced by WOM information (Bansal \& Voyer, 2000). Therefore, in this study, the authors will focus on the seeker-initiated WOM behavior.

A major problem with this kind of WOM communication concerns the sender's response strategies. Considering a high-risk buying situation, which is extremely uncertain in its performance or outcome, the seeker will heavily count on the sender's opinions. However, the sender may hesitate to provide a concrete suggestion for s/he may not be able to confirm both the product's performance and the seeker's preference. The sender might give different WOM message in different situation and different relationship. WOM behavior here reflects the interplay of two overarching social interaction goals: the impression goal and the accuracy goal (Chung \& Darke, 2006). The message giving in this situation is highly adapted to the way the WOM seeker requests information, to the product's category, and to the relationship between WOM dyad. WOM in different context influences the message exchanged between the seeker and the sender, which in turn affects the power of WOM information. Without classifying different types of WOM behavior, we cannot really understand the power of WOM. For researchers and practitioners alike, viewing the WOM behavior as a continuously adaptive interaction between the WOM dyad would be a key step toward a richer theoretical understanding of this complicated and multifaceted phenomenon.

\section{Literature Review}

\subsection{Sender-Initiated WOM}

Previous works have suggested that the main reason for people to spread WOM messages in sender-initiated context mainly because that the consumers' consumption-related expectations 
are disconfirmed (Anderson, 1998). When consumers' consumption-related expectations are disconfirmed, they engage in WOM communication to release their tenseness. That is the reason why people actively spread WOM when they feel surprised or angry. In fact, it could be argued that WOM dispersion is a kind of social sharing of emotions (Derbaix \& Vanhamme, 2003). According to Derbaix and Vanhamme, people will actively share about $90 \%$ of their emotional experiences. Since WOM information is related to personal experience and contains emotional sentences, it can be more vivid and stir response from the receiver that is more emotional (Herr et al., 1991). Therefore, it can have a greater effect on the receivers' buying decision.

Early research had confirmed that opinion leaders (Rogers, 1983) and market mavens (Feick $\&$ Price, 1987) are important WOM senders. These individuals have significant interest in a certain product, actively look for product information, and most importantly, they like to share their knowledge and experience with others. Their motives for spreading WOM information, according to Dichter (1966), could be classified into four categories: product-involvement, self-involvement, other-involvement, and message-involvement. In many cases, people are highly involved in product consumption and actively spread WOM information. Sundaram et al. (1998) conducted 390 critical-incident interviews and identified four motives of consumers to actively spread positive WOM messages; namely, altruism, product involvement, self-enhancement, and helping the company.

\subsection{Seeker-Initiated WOM}

Mangold, Miller, and Brockway (1999) found that seekers' need for information far more likely initiated WOM communication compared to senders' satisfaction level or the marketing organization's promotional efforts. When consumers lack the ability to evaluate a buying decision before making a purchase, they are likely to request the experience of relatives and friends who have already used the product. WOM has proved helpful before a purchase in reducing consumers' uncertainty and assisting customers in comparing alternative services (Bristor, 1990). From this standpoint, consumers who actively seek WOM are searching for a surrogate for direct experience.

Past research confirmed that risk reduction is the main reason for WOM seeking behavior, followed by search time reduction and gathering product information. Consumers also read product-related information on opinion platforms in order to evaluate the product and determine its associated social status (Hennig-Thurau \& Walsh, 2003). However, in seeker-initiated WOM context, it is somewhat unclear if the senders are willing to give their advices about a product. Drawing from exchange theory framework, people will try to defend their self-image (Lin \& Fang, 2006) as well as to show connoisseurship or enhance their image (Hennig-Thurau, et al., 2004). In a high-risk decision task, (for example, "Should I buy this second-hand car?"), the seeker is eager to search for a recommendation; however, the sender may be averse to give advice for s/he is also not sure about the result. In fact, WOM communication is a continuously adjusted process that "reflects the joint influence of an array of information, product, situational, and (inter)personal factors (Chan, 2000)," especially in the seeker-initiated WOM context. 


\section{Methods}

As our major goal is to examine the interaction between the sender and the receiver, we had to collect data from both members of WOM dyad. Gilly et al. (1998) were the first ones to use dyadic WOM data, which they collected via two-step mail surveys; however, they did not inspect the dynamic process of the dyadic data, neither did they compare different perceptions between the seeker and the sender. Since a WOM message may consist of multiple content dimensions, it would be interesting to categorize the types of WOM message in terms of the seekers' request and the senders' strategies in a WOM episode. In accordance with the complexity of WOM interaction in this research, a dyadic in-depth interview method was used to investigate the process and interaction of WOM dyad. Namely, I collected the data from both the seeker and the sender engaged in the same WOM incident. Although, in-depth interview is more expensive and time-consuming compared to the quantitative methodologies, it is considered an appropriate methodology in this study, given the goal of obtaining dynamic and adaptive process of WOM communication. In addition, it is easier to collect paired data by using dyadic in-depth interview.

To conduct in-depth interview, the author followed the process of data collection suggested by Alam (2005). Previous research provides some insight into developing the interview protocol. The interview was conducted in a free-conversation manner, and lasted about 1 hour for each person. After collecting the interview data from the seeker, the senders that the seekers introduced were asked to join our interview. Each interview was audio recorded and then transcribed. Two raters independently analyzed the motivation for the seeker and the sender. The disagreements were resolved through ongoing discussions until a consensus was reached. Overall, 33 interviewees were collected, although only 14 pairs matched.

\section{Results}

\subsection{Seeker's Motivations and Types of Request}

Generally, the literature suggests that the motivations for people to request WOM recommendation are highly consistent. The main reason for people to request WOM is to reduce possible risks. WOM information is considered trustworthier than any other information sources, especially when the sender is believed to have more knowledge, experience, or higher social status. The other two most popular reasons are to accelerate decision-making and to gather more information. The seeker also acquires WOM product-related information in order to evaluate its related social status. In other word, seekers need to know if the product is accepted or appreciated in the view of their friends.

Based on different motivations, WOM seekers engage in different type of request behavior. They might either heavily depend on WOM information, or they might just want to collect more information about the product, or they might simply want to initiate a topic of conversation. The seekers might also differ in the type of information they request. Some people already have a product in mind and ask for a specific product comment or consumption-related experience. However, some might want to obtain more information about a product category in the market and ask for general information to give them direction. 


\section{Macrothink}

The degree of the seekers' information required and WOM dependency can be combined to provide a straightforward model with four types of WOM request behaviors as shown in Figure 1:

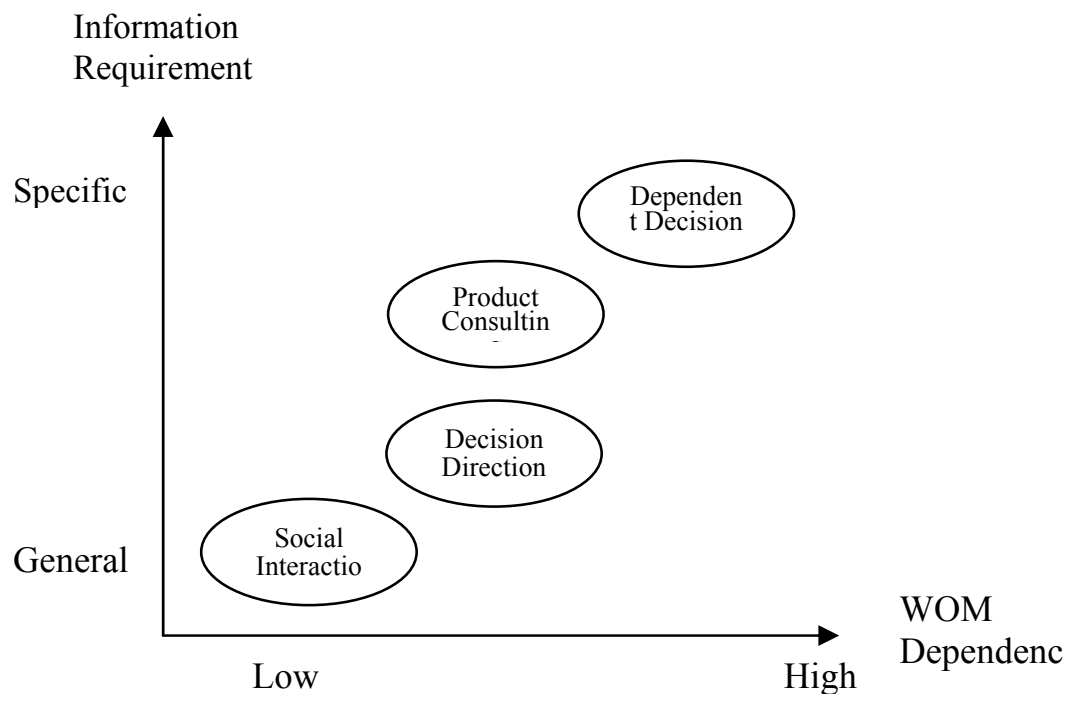

Figure 1. Types of WOM requests

\subsubsection{Dependent Decision}

People sometimes subcontracted their choice task to their friend when they lacked the knowledge to judge the quality of a product. In this situation, the seeker and the sender usually formed a strong relationship. Therefore, the seeker emotionally counted on their friend's judgment to help them make the final decision. As one of our interviewees said, the sender known her (taste and need) very well, and she believed that the sender's suggestion would meet her need. In fact, dependent decision is very rare. Only one case in our data was classified to dependent decision category.

\subsubsection{Decision Direction}

This type of seeker simply wants to know more about the market, for example, where to collect the information or how to make decision, among others. Since seekers are less familiar with the product market than the senders are, WOM information will narrow down the product selection and affect their weight of the product attribute when making the decision.

\subsubsection{Product Consulting}

Some people ask for specific information, including product-specific information (for example, price, where to buy?) and consumption-related experience. People want to know about the product by asking their friends to share their feelings about using the product. They might be willing to buy the product but lack for confidence to make the final decision. As one sender said, "he just needed my support to make sure that he made a good decision." 


\subsubsection{Social Interaction}

Sometimes, WOM exchange is just a topic of their conversation. The seekers initiated a WOM communication, but they did not really want to buy the product. In fact, chatting is the main reason for a seeker to ask a product opinion. The seekers are not purely information receivers; they exchange opinion most of the time.

\subsection{Sender's Motivations}

The major difference between seeker-initiated WOM and sender-initiated WOM is that the WOM receiver is motivated to buy the product that they are currently discussing. Because the sender realizes that his opinion may have great influence on the seeker's final decision, s/he may adopt different strategies in response to the seeker's request. Table 1 presents sender's motivations in seeker-initiated word-of-mouth communication context.

Table 1. Sender's Motivations in Seeker-initiated Context

\begin{tabular}{lll}
\hline Sender's Motivations & Frequency & Percentage \\
\hline Altruism & 14 & $100 \%$ \\
Product involvement & 9 & $64 \%$ \\
Self-enhancement & 9 & $64 \%$ \\
Regret aversion & 9 & $64 \%$ \\
Helping the company & 2 & $14 \%$ \\
Egotism & 1 & $7 \%$ \\
\hline
\end{tabular}

Ccontrary to the past research, the findings revealed two distinct motivations, regret aversion and egotism, but only in seeker-initiated WOM. Regret is a negative emotion that resulted from poor WOM giving. Some people avoid giving concrete product advice when a product is inherently risky. They might respond to the request by providing a general idea, by teaching the seeker to make decisions, or if they do recommend a specific product, by pointing out the drawback of the product in advance. In fact, regret aversion is a very common response in seeker-initiated WOM communication, though it has seldom been addressed in WOM research. Half of the interviewees in this study revealed a tendency to avoid possible regret while giving advice.

WOM communication occurs mainly among people in close relationships. An interviewee (sender) indicated that he recommended the product to his girlfriend because he can borrow the product from her. This type of motivation is viewed as egotism, and it differs from economic motivation. Rosen (2000) mentioned that spreading WOM information has its own economic benefit because the value of a product increases when more and more people consume it (for example MSN or facebook). However, egotism is the direct motivator of purchase linked to the usage of the product, and it seems reasonable when the dyad has a strong relationship, for example, partners or family members.

\subsection{Types of Sender's WOM Comments}

Providing WOM comments to a friend is a highly adaptive behavior. This study proposed three types of WOM comments, which can be explained in figure 2. 


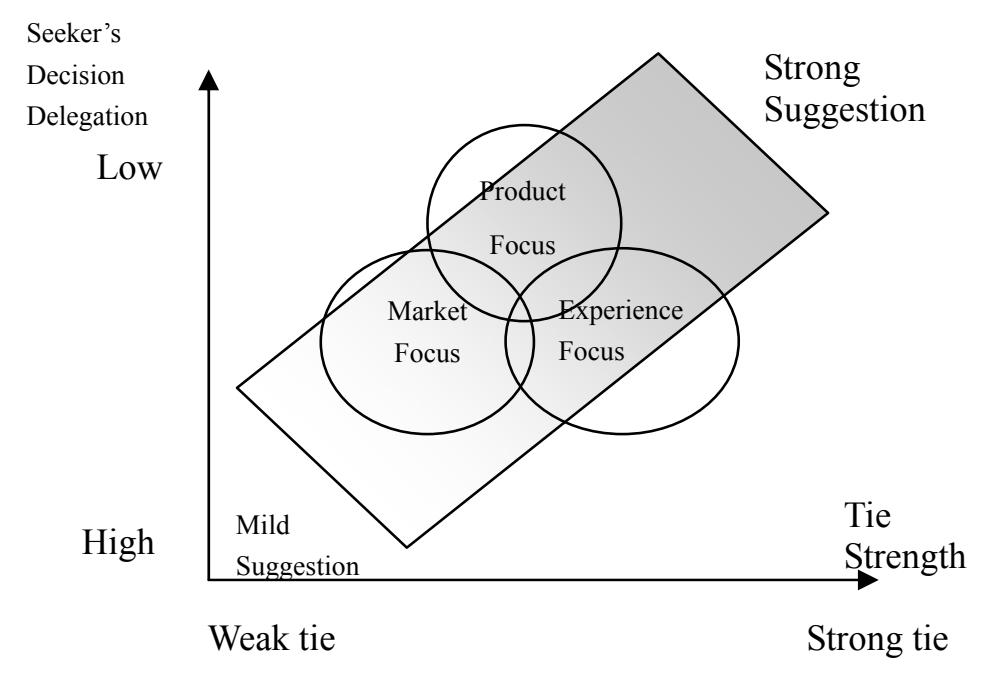

Figure 2. The model of adaptive WOM comments

\subsubsection{Product Focus}

First, some senders provided detail product analysis or detail comparison between alternatives. Most of them were experts or opinion leaders of that product category. They usually provided comments based on objective analysis and tried to help the seeker to compare different product in the market. They acted more like tutors who help the seekers form their decision criteria. From their analysis, the seekers learned the most important attribute of the product and made the decision accordingly. Sometimes, the senders may have suggested the "best buy" for the seeker.

\subsubsection{Market Focus}

The sender's WOM comments focused mainly on sharing price information, product news, and channel evaluation. That is, they provided information about when or where to buy the product. In our data, the senders may have focused on market information because they had just made their own purchase decision (pair 8, 9, 13) or because the sender was in related job position (pair 3).

\subsubsection{Experience Focus}

The senders may have also shared their product-related consumption experience. Their conclusion included their personal experience and feelings and hence was more vivid to the receiver. Although, they also provided product analysis, their suggestion was filled with subjective and emotional sentences. In experience focus type, the senders were more likely to share their WOM comments actively. For example, in pair 8 and 9, the seeker asked for detail information about the service (medical service and mark-up service) because they recalled that the senders once shared their experience with them.

Although, this study proposed three different types of comments, they are not exclusive because the sender exchanges opinions with the seeker in a continuous and dynamic communication process. For example, many people will not only give product news but also 
provide product analysis. People who share their consumption experience might also provide detail market information.

Two important factors, tie strength and the seeker's decision dependency, seem to affect the depth of the sender's WOM comments. As shown in figure 2, the relationship between the seeker and the sender is the most important factor affecting the depth of WOM recommendation. People with close relationship are more involved in their friends' decision and more willing to provide strong suggestions. Some senders even took their friends' purchase decision as their own. For example, in the case of pair 14, the sender helped her sister make a travel decision not only by providing personal comments but also by participating in the entire decision process, collecting information to comparing different travel agents to making the final decision.

Second, some people seem to avoid providing solid suggestions when they realize that the seeker's decision is highly dependent on their comments. For example, in the case of pair 11, the seeker (sister-in-law) asked the sender to make the final decision about a laptop for her, which made the sender feel nervous because he felt total responsibility for her decision. Similarly, in the case of pair 5, the sender avoided to provide solid suggestion about a second-hand car for the seeker.

\section{Discussions and Conclusion}

This research investigates the adaptive motivations of WOM communication in the seeker-initiated context. By applying the dyadic in-depth interviewing method, the author can directly examine the patterns of seeker-initiated WOM communication. Overall, the results of this study triggered us to reconsider WOM communication as a highly adaptive behavior. The senders adapted their recommendations according to the seekers' request styles. Unlike the results found in previous research (Hennig-Thurau \& Walsh, 2003), motivations for people to spread WOM in response to the seeker's request is very different from that of it being actively spread. Risk aversion and egotism are two unique motivations only found in the seeker-initiated context. People used different strategies to reduce possible feelings of regret, or they might have tried to benefit from their friends' purchase decision by suggesting the product they themselves want.

When the sender is aware of the high cost of an unsuitable recommendation, risk aversion motivation will dominate the communication. Some senders have tried to avoid possible regret from unsuitable WOM recommendations and used different strategies to provide their opinion without initiating a solid suggestion. First, they might have suggested various products with different pros and cons. The seekers had to do more research before making their own decision. Second, they might have taught the seeker how to make decision. In other words, they tried to provide a decision rule for the seeker. Third, they might have just provided objective information without pointing out which product might be the best. Finally, they might have just shared their experience, including good and bad side of the product. No matter what strategies they used, they all encouraged the seeker to do more research prior to making a final decision. 
Since WOM communication is a highly adaptive behavior, the sender will adjust his/her WOM comments in accordance with different request styles made by the seeker. In this study, four types of WOM requests: dependency decision, decision direction, product consulting and social interaction, are proposed. It seems that all senders respond to different WOM requests in a very similar manner. The sender feels free to share personal experience and product comments when the main goal of communication is social interaction. The sender avoids possible regret from WOM giving when the seeker greatly depends on his/her judgment. The depth of WOM comments, as I call the "referral laddering," is contingent on both personal factors (e.g., expertise, perceived risk, opinion leadership, age) and interpersonal factors (e.g., tie strength, decision dependency). This study is presenting the preliminary results of this issue that requires further investigation to be expanded into a more comprehensive model.

\section{Limitations and Directions for Further Research}

Although this study offers a fresh perspective on WOM behavior, several limitations and future research suggestions deserve mentioning. First, given the preliminary nature of the study, the study's samples may be biased in that the study collected data chosen from a small-scale convenient sampling method. Moreover, to conduct the dyadic analysis, the WOM sender was limited to only one person and was introduced by the seeker. This method simplified each WOM episode to one-to-one communication; however, in reality, most people seek decision assistance from more than one person when the decision is risky. The simplification is helpful when trying to analyze dyadic data, but future research may focus on the integration of WOM into a multi-communication model based on this research investigation.

Second, the study used retrospective recall to collect the WOM episode. Thus, interviewees had a tendency to recall the most memorable and most recent incidents. Although the author used different strategies to encourage interviewees to recall as many WOM seeking situations as possible, it is important to recognize that the retrospective method may not perfectly produce the most typical incidents. Since the study didn't adequately control WOM incidents to a specific product, the variance result from different product types may be high. Future research is obviously required, but this is an exciting first step.

Third, since WOM communication is a highly culturally related behavior (Money, Gilly, \& Graham, 1998; Mooradian \& Swan, 2006), it is extremely possible that the interaction patterns between the dyad will be somewhat different in comparison with collectivism and individualism culture. For example, past research found that the Japanese have an extremely risk-averse society compared to Americans (Hofstede, 1991). Hence, it is reasonable to assume that the benefits and costs of giving recommendations are potentially higher in other-focus society than those in a self-focus society. Future research will hopefully clarify this important issue. 


\section{Acknowledgement}

The authors gratefully acknowledge financial support from the National Science Council in Taiwan. (NSC 96-2416-H-231-010)

\section{References}

Aggarwal, P., \& Mazumdar, T. (2008). Decision delegation: A conceptualization and empirical investigation. Psychology \& Marketing, 25(1), 71-93. http://dx.doi.org/10.1002/mar.20201

Alam, I. (2005). Fieldwork and Data Collection in Qualitative Marketing Research. Qualitative Market Research, 8(1), 97-112. http://dx.doi.org/10.1108/13522750510575462

Anderson, E. W. (1998). Customer Satisfaction and Word-of-Mouth. Journal of Service Marketing, 1(1), 5-17.

Bansal, H. S., \& Voyer, P. A. (2000). Word-of-Mouth Processes Within a Services Purchase Decision Context. Journal of Service Research, 3(2), 166-177. ttp://dx.doi.org/10.1177/109467050032005

Bristor, J. M. (1990). Enhanced Explanations of Word of Mouth Communications: The Power of Relationships. Research in Consumer Behavior, 4, 51-83.

Chan, H. (2000). Adaptive Word-of-Mouth Behavior: A Conceptual Framework and Empirical Tests. Unpublished Business Thesis, University of Wisconsin-Madison.

Chung, C., \& Darke, P. (2006). The consumer as advocate: Self-relevance, culture, and word-of-mouth. Marketing Letters, 17(4), 269-279. http://dx.doi.org/10.1007/s11002-006-8426-7

Cohen, A. (1999). A Best-Seller by Word-of-Mouth. Sales and Marketing Management, 151, 15.

Derbaix, C., \& Vanhamme, J. (2003). Inducing word-of-mouth by eliciting surprise - a pilot investigation. Journal of Economic Psychology, 24, 99-116. ttp://dx.doi.org/10.1016/S0167-4870(02)00157-5

Feick, L. F., \& Price, L. (1987). The Market Maven: A Diffuser of Marketplace Information. Journal of Marketing, 51(1), 83-97. http://dx.doi.org/10.2307/1251146

Gilly, M., Graham, J., Wolfinbarger, M., \& Yale, L. (1998). A dyadic study of interpersonal information search. Journal of the Academy of Marketing Science, 26, 83-100. http://dx.doi.org/10.1177/0092070398262001

Hennig-Thurau, T., \& Walsh, G. (2003). Electronic Word-of-Mouth: Motives for and Consequences of Reading Customer Articulations on the Internet. International Journal of Electronic Commerce, 8(2), 51-74.

Hennig-Thurau, T., Gwinner, K. P., Walsh, G., \& Gremler, D. D. (2004). Electronic Word-of-Mouth Via Consumer-Opinion Platforms: What Motivates Consumers to Articulate 


\section{Macrothink}

Themselves on the Internet? Journal of Interactive Marketing, 18(1), 38-52. http://dx.doi.org/10.1002/dir.10073

Herr, P. M., Kardes, F. R., \& Kim, J. (1991). Effects of Word-of-Mouth and Product-Attribute Information of Persuasion: An Accessibility-Diagnosticity Perspective. Journal of Consumer Research, 17(4), 454-462. http://dx.doi.org/10.1086/208570

Hofstede G (1991). Cultures and Organizations-software of the mind. London; McGraw-Hill.

Lin, T. M. Y., \& Fang, C.-H. (2006). The Effect of Perceived Risk on the Word-of-Mouth Communication Dyad. Social Behavior and Personality, 34(10), 1027-1216. http://dx.doi.org/10.2224/sbp.2006.34.10.1207

Mangold, W. G., Miller, F., \& Brockway, G. R. (1999). Word-of-mouth communication in the service marketplace. Journal of Services Marketing, 13(1), 73-89. http://dx.doi.org/10.1108/08876049910256186

Money R. B., Gilly M. C., \& Graham J.L. (1998). Explorations of national culture and word-of-mouth referral behavior in the purchase of industrial services in the United States and Japan. Journal of Marketing, 62(4), 76-88. http://dx.doi.org/10.2307/1252288

Mooradian T. A., \& Swan K. S. (2006). Personality-and-culture: The case of national extraversion and word-of-mouth. Journal of Business Research, 59(6), 778-785. http://dx.doi.org/10.1016/j.jbusres.2006.01.015

Rogers, E. M. (1983). Diffusion of Innovations (3th ed.). New York: Free Press.

Rosen, E. (2000). The Anatomy of Buzz: How to Create Word-of-Mouth Marketing. New York: Doubleday.

Silverman, G. (2001). The Secrets of Word-of-Mouth Marketing: How to Trigger Exponential Sales Through Runaway Word of Mouth. NY: American Management Association.

Sundaram, D. S., Mitra, K., \& Webster, C. (1998). Word-of-Mouth Communications: A Motivational Analysis. Advances in Consumer Research, 25(1), 527-531. 